\title{
Research and design of small and medium power without the impact of the UPS
}

\author{
WANG Yong \\ (Hunan Railway Professional Technology College, ZhuZhou Hunan 412001)
}

Keywords: Electric vehicle; power battery; photovoltaic battery; charging device.

\begin{abstract}
The design for the normal operation of load in a period of time when the electricity suddenly interrupted. The whole design used "AC-DC-AC" form. The direct current can be obtained by the electricity after the rectifier or the battery voltage after boosting and then sent out after boosting and inverter. The cutting process can be realized by diode using voltage differences between the two DC power supply modes. The cutting process will not produce any phenomenon of phase differences and the instantaneous power outages and will not need the involvement of software and detection circuit. The cutting process has highly reliability, lower fluctuation of the AC voltage and surge-free.
\end{abstract}

\section{Introduction}

Currently, to ensure the normal operation of information security and computer systems, automated assembly line and communication systems, power supply quality requirements more demanding. In important electricity sectors, such as banking, aviation, military security and quality of these industries supply requirements are getting higher and higher, unprotected power system may cause hardware damage, data loss or business interruption caused by abnormal , may cause significant damage equipment or personnel. Therefore, high reliability, research and development of non-impact uninterruptible power supply becomes more and more important, it uses "AC - DC AC" form, designed a medium power switching without shocks UPS, switching process utilizes two the voltage difference between the kinds of DC power supply, with the diodes, switching process does not produce any phase and instantaneous blackouts, tested test, the UPS high reliability, small voltage fluctuation AC side, no impact.

\section{System Design}

\subsection{Overall system design}

When the mains is normal, the battery does not provide power to the inverter by hardware shutdown this channel; by level of Boost circuit, sine wave inverter output after filtering by filter supplied to the load. When utility power fails or the power quality mains UPS is outside the scope of the requirements, the rectifier bridge to stop working, after two battery output voltage of Boost circuit voltage raised to just below the single-stage Boost output voltage, the inverter begin to provide energy load. When the output short circuit or battery voltage below the permissible value, UPS to stop working, to prevent damage to the inverter or battery. When the output overload, if the overload is instantaneous, it can be controlled to allow this to happen, if you overload a long time, then you need to switch the UPS to the mains supply to the load.

\subsection{The main circuit topology design}

220V single-phase AC power supply input; simple rectifier selection structure, high power factor of the diode bridge rectifier; inverter system based in part on the capacity of the switching frequency level of difficulty and other factors driving circuit into account, select MOSFET as an inverter bridge switching devices. Rectified output voltage is only $280 \mathrm{~V}, 220 \mathrm{~V}$ AC can not be achieved after the inverter, so uplifted by a single-stage Boost circuit to 300V. Battery output voltage is only $12 \mathrm{~V}$, so the use of two series circuit Boost voltage raised to $270 \mathrm{~V}, 220 \mathrm{~V}$ AC output via the inverter. Main circuit topology shown in Figure 2. Figure, C1, C2, C3 of large capacitance, played the role of filter and regulator. 


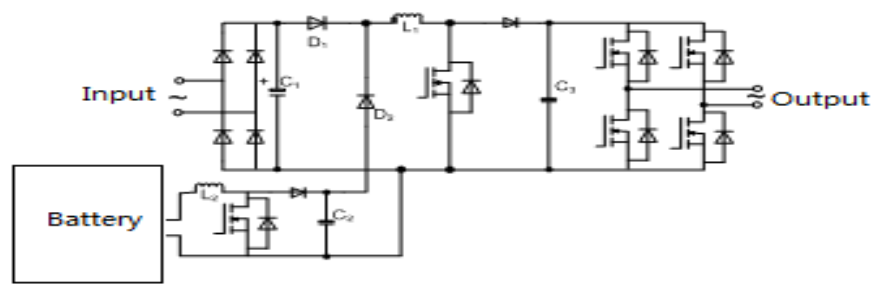

Figure 1 main circuit topology

The system uses a single-phase full-bridge uncontrolled rectifier bridge and a single full-bridge inverter, DC / DC achieve boost, which can reduce the complexity of the control.AC / DC using non-controlled rectifier, single phase mains to DC rectifier.DC / DC Boost circuit is completed using two boost,respectively rectified output voltage and the battery output voltage is raised to a higher current, so that the rear stage inverter output is

$220 \mathrm{~V} / 50 \mathrm{~Hz}$ single-phase AC power.DC / AC single-phase inverter part of the whole bridge, the role of direct current into $220 \mathrm{~V} / 50 \mathrm{Hzsine}$ AC power to the load to achieve the inverter.

\subsection{Parameter Design}

\subsubsection{Output Boost rectifier circuit parameters}

Figure 2 is rectified output Boost converter circuit structure.

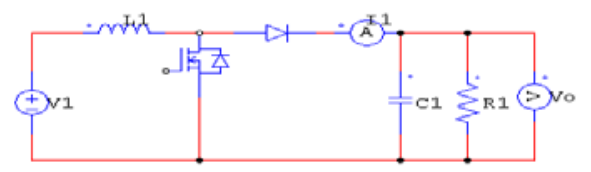

Figure 2 circuit structure of the Boost converter

Boost converter input rectifier output single-phase uncontrolled rectifier output mains, ranging $\mathrm{V} 1=280 \mathrm{~V}$, output voltage $\mathrm{VO}=300 \mathrm{~V}$, then according to the formula

$$
\frac{U_{O}}{U_{i}}=\frac{1+\sqrt{1+\frac{4}{K}}}{2}
$$

Calculated $\mathrm{D}=0.075$, by the formula

$$
\begin{gathered}
C_{1}=\frac{I_{0} \times\left(V_{0}-V_{1}\right)}{f_{s} \times V_{0} \times \Delta V_{0}} \\
L_{1}=\frac{V_{0} \times D \times(1-D)^{2}}{2 \times f_{s} \times V_{0}}
\end{gathered}
$$

$C_{1}=400 \mu F, L_{1}=1.1 \mathrm{mH}$ 。 And $C_{1}=470 \mu F, L_{1}=6.2 m H_{\circ}$

\subsubsection{Parameter design of Boost circuit for storage battery}

Figure 3 circuit structure for the storage battery Boost converter.

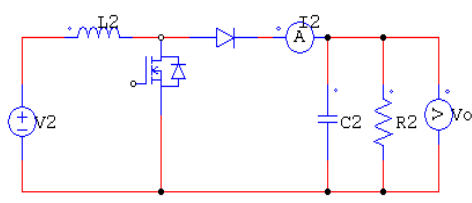

Figure 3 circuit structure of the battery Boost converter

The input voltage of Boost converter is connected with the series output voltage of two batteries, the value of $\mathrm{V} 2=36 \mathrm{~V}$, the Boost of the battery is $\mathrm{VO}=270 \mathrm{~V}$. 
The inductance current of the battery Boost is continuous, so according to the formula

$$
\frac{V_{O}}{V_{2}}=\frac{1}{1-D}
$$

Can be calculated from the second levels of the Boost D2 $=0.867$.

Then according to the formula (2), (3) F C2 $=120, \mathrm{~L} 2=0.8 \mathrm{mH}$. The actual selection of $\mathrm{C} 2=470 \mathrm{~F}$, $\mathrm{L} 2=6.2 \mathrm{mH}$.

\subsubsection{Switching tube absorption parameters in DC/DC circuit}

$\mathrm{DC} / \mathrm{DC}$ circuit in the switch tube are the use of RCD filter, $\mathrm{C}=0.1 \mathrm{~F}, \mathrm{R}=1.5 \mathrm{~K}(1 / 2 \mathrm{~W}), \mathrm{D}$ reverse voltage $600 \mathrm{~V}$ to reverse the rapid recovery diode.

\section{Hardware circuit design}

\subsection{System main circuit}

According to the design idea of the system, the main circuit of the UPS power system can be obtained as shown in Figure 4.

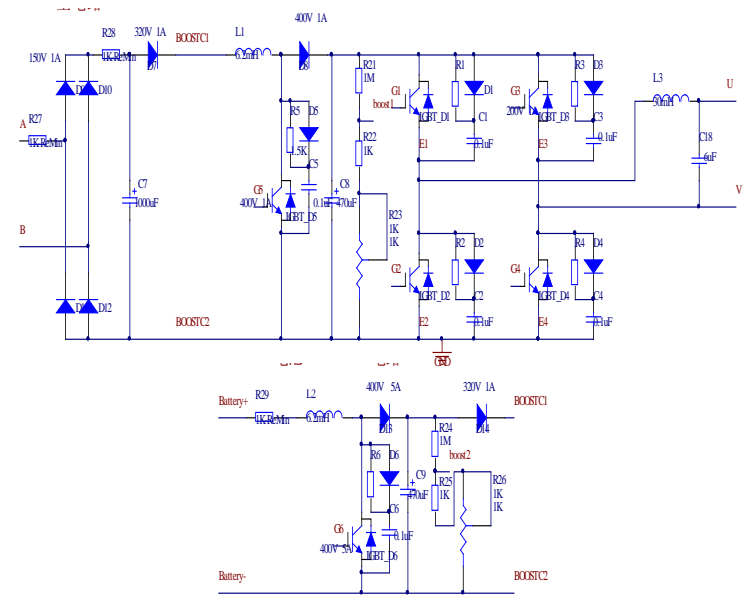

Figure 4 main circuit of UPS power supply system

\subsection{Inverter circuit IGBT drive circuit}

Two chip IR2110 chip, the driver circuit is shown in figure 5:

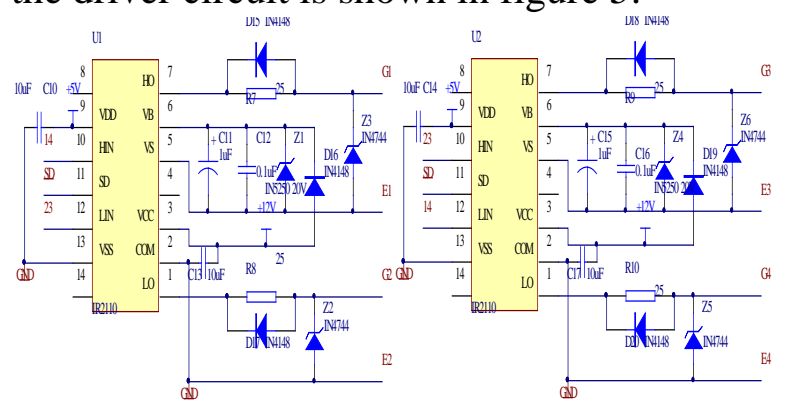

Figure 5 inverter circuit MOSFET drive circuit

$\overline{S D}$ is a PWM pulse blocking signal, 23, two respectively for a bridge arm up and down 14 IGBT drive signal. DSP output PWM is converted to $0-15 \mathrm{~V}$ by 7407 OC, thus meeting the requirements of IR2110.

\subsection{DC/DC converter circuit IGBT driver circuit}

The transistor is driven by a transistor, and the transistor is chosen for C9013, and the driving circuit is shown in Figure 6. 


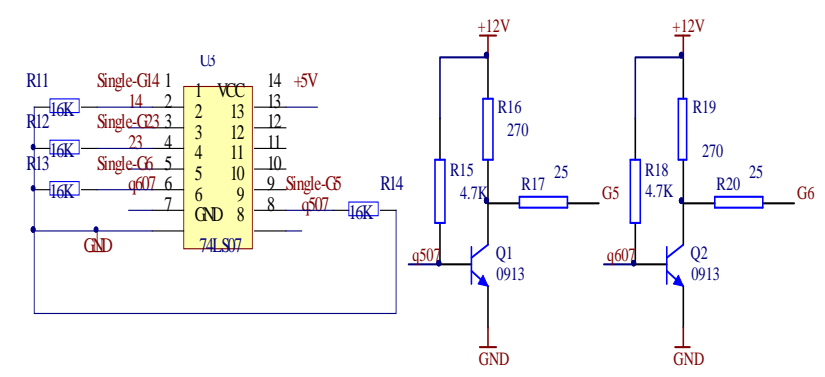

Figure 6 DC/DC converter circuit MOSFET drive circuit

Single-G5, q507, G5, Single-G6, G6, MOSFET, and q607 switching devices are the driving signals of the two channels of the converter DC/DC. DSP output PWM is converted to $0-15 \mathrm{~V}$ by 7407 OC, thus meeting the requirements of the C9013 input.

\section{System software design}

Repetitive control is to design a controller to ensure that the error of the system is zero. For the UPS inverter control system, its instruction is sinusoidal variation, and the load current will change according to the change of the load, when the line is connected to the load show that the current sinusoidal variation, connected with nonlinear loads, a non sinusoidal variation, according to the traditional PID control, according to the known principles of endometrium, control effect is poor.

For the problem of the non static error tracking of the sinusoidal instruction, a sinusoidal signal model with the same frequency as the instruction can be implanted into the controller:

$$
C_{m}(s)=\frac{\omega}{s^{2}+\omega^{2}}
$$

It can be verified that when both the instruction and the load current are sinusoidal at the angular frequency, according to the inner membrane principle, the closed-loop system with a stable inner membrane is not static. But in practical application, often containing multiple nonlinear loads, the load current with multiple harmonics, if you want to all of the load current are no static error, the general practice is to set a membrane for each harmonic, but to do so would be the complex structure of the system and it is difficult to achieve. Taking into account the load current in each of the fundamental cycle to repeat the same waveform, this design uses the "repeat signal generator" intima:

$$
G_{m}(s)=\frac{1}{1-e^{-L S}}
$$

Where L for inverter output fundamental cycle. This is a cycle delay by a positive feedback loop, irrespective of the input signal, as long as the fundamental cycle repeated, the output of the model is the input signal by cycle accumulation.

When such a link is placed in the forward channel of the feedback control system, it plays a role in the process of the integration and is similar to the integral part. A repeat signal generator is an accumulation of the error in the period of the cycle. If the system is stable, it can achieve zero steady-state error. Because of the delay in the repetitive control signal generator, it is not easy to simulate, so the actual system is usually achieved by numerical method:

$$
G_{m}(z)=\frac{1}{1-z^{-K}}
$$

Which $\mathrm{K}$ is the number of times per fundamental frequency.

The repetitive signal generator can be regarded as the time step

The pure integrator, it although in theory there is no static error can be realized, but the stability and the robustness of the adverse, because it is equivalent to the system to bring the $\mathrm{k}$ a unit circle on the open-loop poles, system parameters slightly changes or modeling a slight error will lead to the loss of stability of the closed-loop system. So the actual system uses the improved repetitive signal generator. Figure 7 is a commonly used improvement plan: direct repetitive control scheme. 


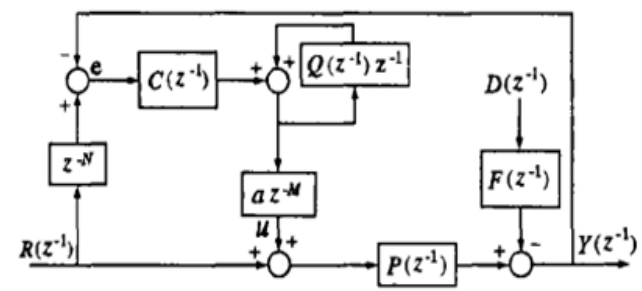

Figure 7 improved repeat control algorithm block diagram

\section{Test}

\subsection{Experimental waveforms of the power supply to the load}

After rectification and filtering of the city, through the Boost circuit, the rectifier output voltage will be raised to a higher voltage, the inverter is carried out, and the AC current of $220 \mathrm{~V} / 50 \mathrm{~Hz}$ is obtained.

Figure 8 shows the power of the city, the city after the rectification and Boost boost, the AC waveform obtained by the inverter. Because of the reason of the voltage probe of the oscilloscope, only half of the waveform can be found

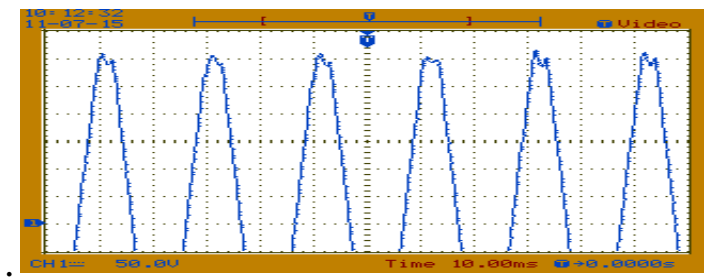

Figure 8 inverter output voltage when power supply

\subsection{Experimental waveforms of battery on load power supply}

The battery output through the two level Boost circuit, the rectifier output voltage is raised to a higher voltage, the inverter is carried out, and the $220 \mathrm{~V} / 50 \mathrm{~Hz} \mathrm{AC}$.

Figure 9 shows the battery, the battery output voltage by two Boost after the boost, through the inverter AC waveform.

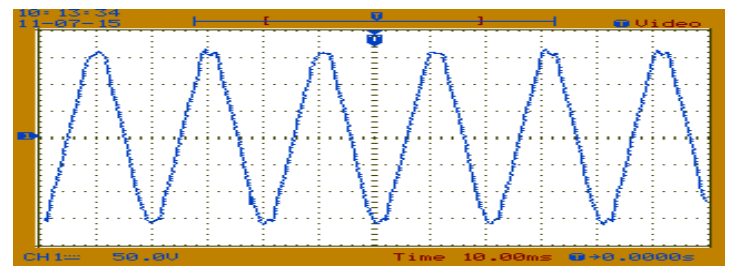

Figure 9 inverter output voltage when battery power supply

\section{Conclusion}

The design method of "AC - DC - AC" integrated design method, the DC power from the city of electric rectifier or battery boost, and then through the boost and inverter. Switching process using two DC power supply voltage difference, implementation using diode, switching process does not produce any phase difference and instantaneous interruption phenomenon, and without the intervention of the detection circuit and software, through the test and test, high reliability, small fluctuations in the voltage of AC side, no impact.

\section{Papers Support Fund:}

Hunan Provincial Department of Education teaching science research projects ( numbers: 14C0757). 


\section{References}

[1] MAI Yan-Hong. Mobile platform design based on automatic lifting [J]. Scientific practice. 2013 12:317.

[2] YU Wei. Research on some key problems of UPS parallel system [D]. Doctoral Dissertation of Zhejiang University. 2009.

[3] TANG Xiang-Xin. Research on the key technologies of UPS digital control and parallel operation [D]. Master degree thesis of South China University of Technology.2010.

[4] LU Xiao-Hong. Research on control technology of UPS inverter [D]. Master degree thesis of Dalian University of Technology.2005.

[7]ZHANG Zheng. Design and implementation of UPS monitoring software system [D]. Master degree thesis of Ocean University of China. 2007.

[6]ZOU Jie. Research of online UPS power supply based on DSP [D]. Master degree thesis of Daqing Petroleum Institute. 2009. 